\title{
Photoionization of Clustered Halos by the First Stars
}

\author{
Daniel Whalen*, Brian W. O’Shea ${ }^{\dagger}$, Joseph Smidt** and Michael L. Norman ${ }^{*}$ \\ *Applied Physics (X-2), Los Alamos National Laboratory, Los Alamos, NM 87545, U.S.A. \\ ${ }^{\dagger}$ Theoretical Astrophysics (T-6), Los Alamos National Laboratory, Los Alamos, NM 87545, U.S.A. \\ ${ }^{* *}$ Department of Physics and Astronomy, Brigham Young University, Provo, UT 84602, U.S.A. \\ ${ }^{\ddagger}$ Center for Astrophysics and Space Sciences, University of California at San Diego, La Jolla, CA 92093, U.S.A.
}

\begin{abstract}
.
We present numerical simulations of the photoevaporation of cosmological halos clustered around a $120 \mathrm{M}_{\odot}$ primordial star, confining our study to structures capable of hosting Population III star formation. The calculations include self-consistent multifrequency conservative transfer of UV photons together with nine-species primordial chemistry and all relevant radiative processes. The ultimate fates of these halos varies with central density and proximity to the central source but generally fall into one of four categories. Diffuse halos with central densities below $2-3 \mathrm{~cm}^{-3}$ are completely ionized and evaporated by the central star anywhere in the cluster. More evolved halo cores at densities above $2000 \mathrm{~cm}^{-3}$ are impervious to both ionizing and Lyman-Werner flux at most distances from the star and collapse of their cores proceeds without delay. Radiative feedback in halos of intermediate density can be either positive or negative, depending on how the I-front remnant shock both compresses and deforms the core and enriches it with $\mathrm{H}_{2}$. We find that the $120 \mathrm{M}_{\odot}$ star photodissociates $\mathrm{H}_{2}$ in most halos within the cluster but that catalysis by $\mathrm{H}$ - rapidly restores molecular hydrogen within a few hundred Kyr after the death of the star, with little delay in star formation. Our models exhibit significant departures from previous one-dimensional spherically-symmetric simulations, which are prone to serious errors due to unphysical geometric focusing effects.
\end{abstract}

Keywords: Stars: formation; HII regions; large-scale structure of the universe PACS: $97.20 . \mathrm{Wt}, 98.80-\mathrm{k}$

\section{INTRODUCTION}

Radiative feedback from one generation of stars on the next regulated the rise of stellar populations on many spatial scales since the highest redshifts. In early times this feedback came in two basic forms: ionizing UV photons and Lyman-Werner (LW) radiation between 11.18 $\mathrm{eV}$ and $13.6 \mathrm{eV}$ that photodissociates molecular hydrogen. Very massive primordial stars photoionized other cosmological halos clustered in their vicinity, in many instances preventing their collapse. It is also believed that the H II regions of the first stars and protogalaxies established an "entropy floor" with elevated IGM temperatures that discouraged new star formation in general [8]. Prodigous sources of ionizing UV also emitted copious numbers of LW photons capable of sterilizing nearby halos of $\mathrm{H}_{2}$, the key coolant permitting primordial gas to condense and form the first stars. These photons propagated to much greater distances than ionizing UV because the IGM was transparent to them. Over time this photodissociating background grew, permeating the early cosmos and suppressing $\mathrm{H}_{2}$ formation in the first pregalactic objects. These are three examples of negative radiative feedback. On the other hand, molecular hydrogen can form in the outer layers of primordial I-fronts that later enhances cooling in the IGM [12], and such fronts can drive shocks into nearby cosmological halos, perturbing their cores and accelerating their collapse into stars. How these two types of feedback competed to either promote or suppress subsequent star formation remains unclear.

\section{Numerical Models of Radiative Feedback}

Computer simulations of radiative feedback on star formation fall into three categories. Those performed in cosmological simulation volumes $\left(1 \mathrm{Mpc}^{3}\right.$ or more) follow large-scale structure formation without radiative transfer, treating both ionizing and LW photons as a uniform background. These studies suggest that metagalactic dissociating fluxes postpone but do not prevent primordial cloud collapse into stars [6, 7, 11, 20]. The second type are models that follow star formation on small scales in cosmological halos proximate to the first stars [10]. These surveys also sacrifice radiation transport to follow the detailed collapse of the halo into a new star. Radiation is observed to exert both positive and negative feedback on primordial cloud collapse in these studies. More ambitious campaigns attempt to assemble the first primeval galaxies by the consecutive formation of primordial stars, one often in the relic $\mathrm{H}$ II region of its predecessor [5, 21]. In these calculations, which emphasize radiative feedback in protogalaxy evolution, star formation in remnant ionized fields is weakly suppressed and, due to HD cooling, leads to a less massive generation of 
stars.

Simulations in the third and most recent category focus on the dissociation and evaporation of the halo rather than its collapse into a star, inferring the likelihood of star formation from its final state [14, 13, 1, 2]. These calculations find a variety of outcomes to halo photoevaporation, ranging from flash ionization and complete eviction of baryons from diffuse halos to insulation from any radiation effects whatsoever in dense cores (collapse of the halo proceeds unimpeded in such cases). Star formation in cosmological halos of intermediate densities can be either pre-empted or accelerated. The most comprehensive survey to date was by Ahn \& Shapiro [2], who found local radiative feedback to be mostly neutral. Unfortunately, this well-parametrized study is prone to unphysical geometrical focusing in its 1D Lagrangian grid, which leads to serious departures from the true evolution of the halos.

\section{CODE ALGORITHM/MODEL PARAMETERS}

We performed a suite of two-dimensional axisymmetric radiation hydrodynamical calculations of the ionization and photodissociation of a cosmological minihalo by a $120 \mathrm{M}_{\odot}$ star [18]. The halo is taken from an Enzo AMR code1 [9] simulation and spherically-averaged. The halo is then imported into the ZEUS-MP code 2 and photoevaporated. ZEUS-MP [15, 16] is a massively-parallel Eulerian reactive flow hydrocode with self-consistent multifrequency photon-conserving UV radiative transfer [17] coupled to nine-species primordial gas chemistry [3].

The four evolutionary stages of the $1.35 \times 10^{5} \mathrm{M}_{\odot}$ primordial halo considered in our study are shown in Fig 1. This halo mass was selected because it is the smallest in which a star would be expected to form. Since the ionization front would have less impact on more massive halos, feedback effects would be most pronounced in this one. Its central densities range from $1.43 \mathrm{~cm}^{-3}$ to 1596 $\mathrm{cm}^{-3}$ from $z=23.9$ to 15.0 . We consider consecutive profiles from a single halo rather than sampling the entire cluster at a fixed redshift for three reasons. First, the halos in the cluster have similar profiles and are largely coeval, so the emergent I-front may encounter a narrower range of density gradients at a single redshift than in one halo over a range of redshifts. Second, the time at which the cluster is engulfed by the expanding $\mathrm{H}$ II region is an open parameter. Finally, we avoid constraining our results to a single cluster of halos and its associated

1 http://lca.ucsd.edu/portal/software/enzo

2 http://lca.ucsd.edu/portal/software/zeus-mp2

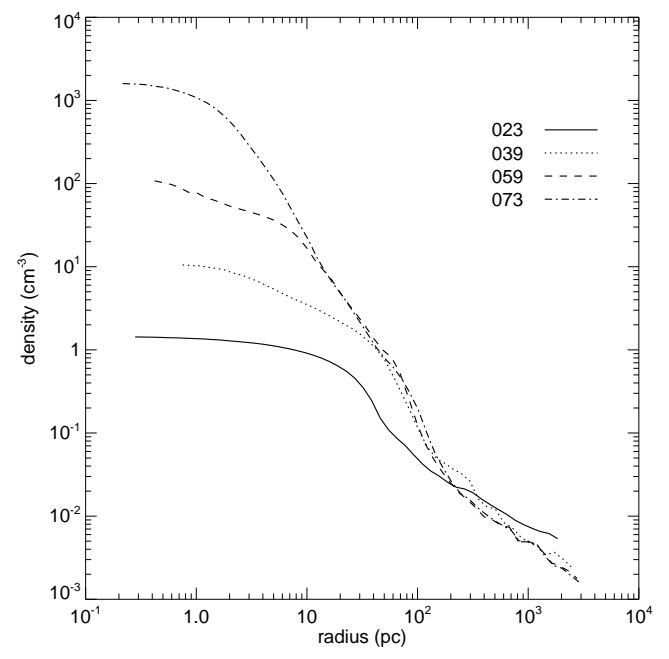

FIGURE 1. The four halo profiles. The redshifts of the 023, 039, 059, and 073 profiles are 23.9, 17.7, 15.6, and 15.0, respectively.

properties (number of peaks, diameter, etc). The range of radiative feedback in the entire cluster is better explored by evaporating a single halo whose central densities vary from low values that are easily ionized to high values in which core collapse would proceed uninterrupted. Each profile was illuminated by a plane wave of photons from a $120 \mathrm{M}_{\odot}$ star at four distances: $150,250,500$, and 1000 pc. The plane wave was geometrically attenuated by $1 / R^{2}$, where $R$ is the distance of the I-front from the star. The cloud is irradiated for $2.5 \mathrm{Myr}$, the main sequence lifetime of the $120 \mathrm{M}_{\odot}$ star, and then left to evolve in the relic H II region an additional 2.5 Myr. As a control we chemothermally evolved the halo for $4.8 \mathrm{Myr}$ without radiation to determine the central densities to which the core condenses in the absence of stellar feedback. Core densities in the 023 and 039 halos change by $\sim 1 \%$, those in the 059 halo rise from $108 \mathrm{~cm}^{-3}$ to $200 \mathrm{~cm}^{-3}$, and those in the 073 halo increase from $1600 \mathrm{~cm}^{-3}$ to 6000 $\mathrm{cm}^{-3}$.

\section{FIDUCIAL CASE}

We centered the 073 halo at the origin of a twodimensional axisymmetric cylindrical coordinate box, with boundaries of $-125 \mathrm{pc}$ and $125 \mathrm{pc}$ in $z$ and 0.01 $\mathrm{pc}$ and $125 \mathrm{pc}$ in $r$. The grid was discretized into 1000 zones in $z$ and 500 zones in $r$ for a spatial resolution of $0.25 \mathrm{pc}$. Outflow conditions were applied to the upper and lower $z$ boundaries and reflecting and outflow conditions were assigned to the inner and outer boundaries in $r$, respectively. The gas was primordial, $76 \% \mathrm{H}$ and $24 \% \mathrm{He}$ by mass. The gravitational potential of the dark 

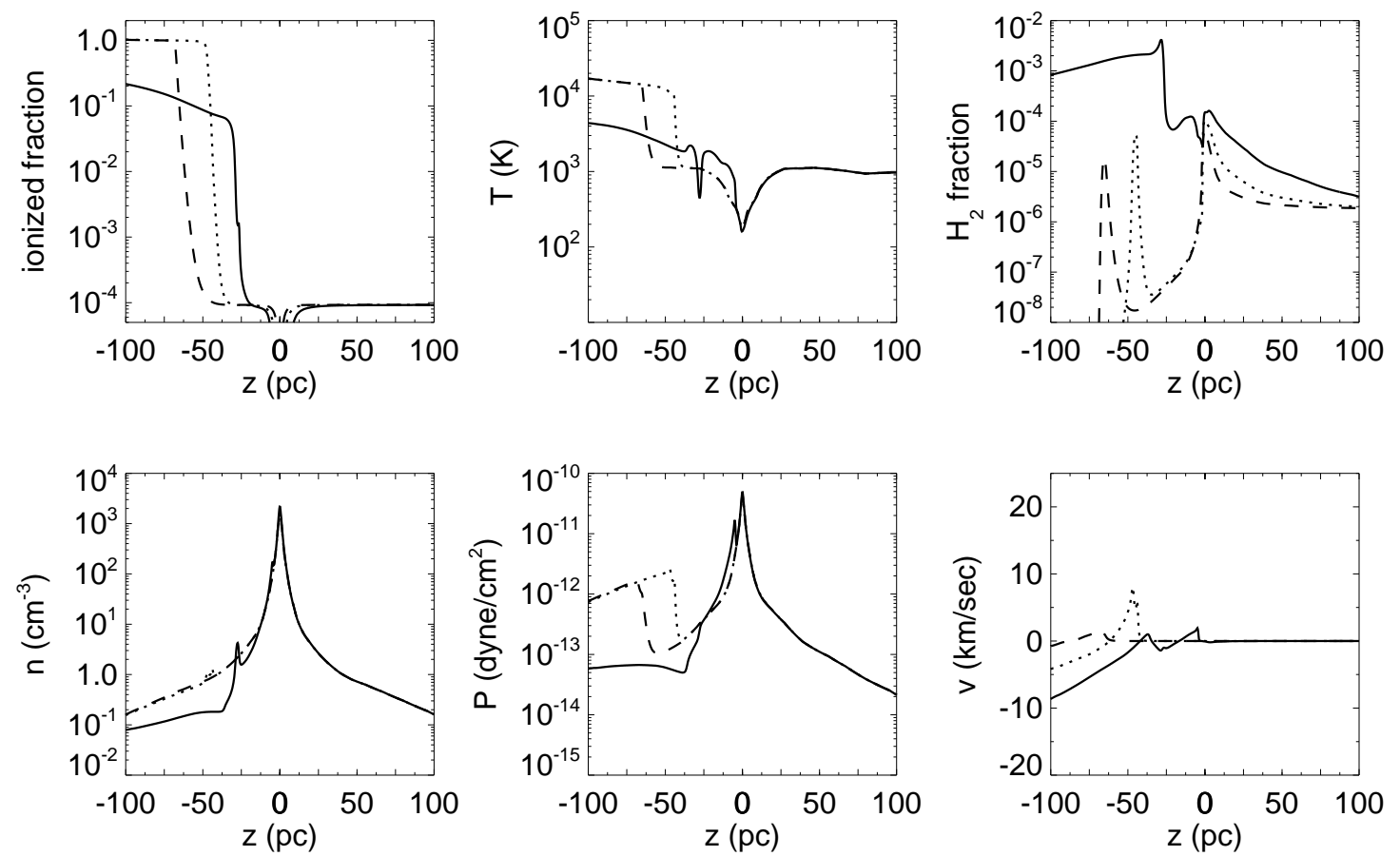

FIGURE 2. Ionized fraction, density, temperature, pressure, $\mathrm{H}_{2}$ fraction and velocity profiles through the center of the 073 halo $500 \mathrm{pc}$ from the star. Dashed line: $200 \mathrm{kyr}$ (the R-type front), dotted line: $800 \mathrm{kyr}$ (the D-type front), solid line: $5.0 \mathrm{Myr}$ (the relic H II region).

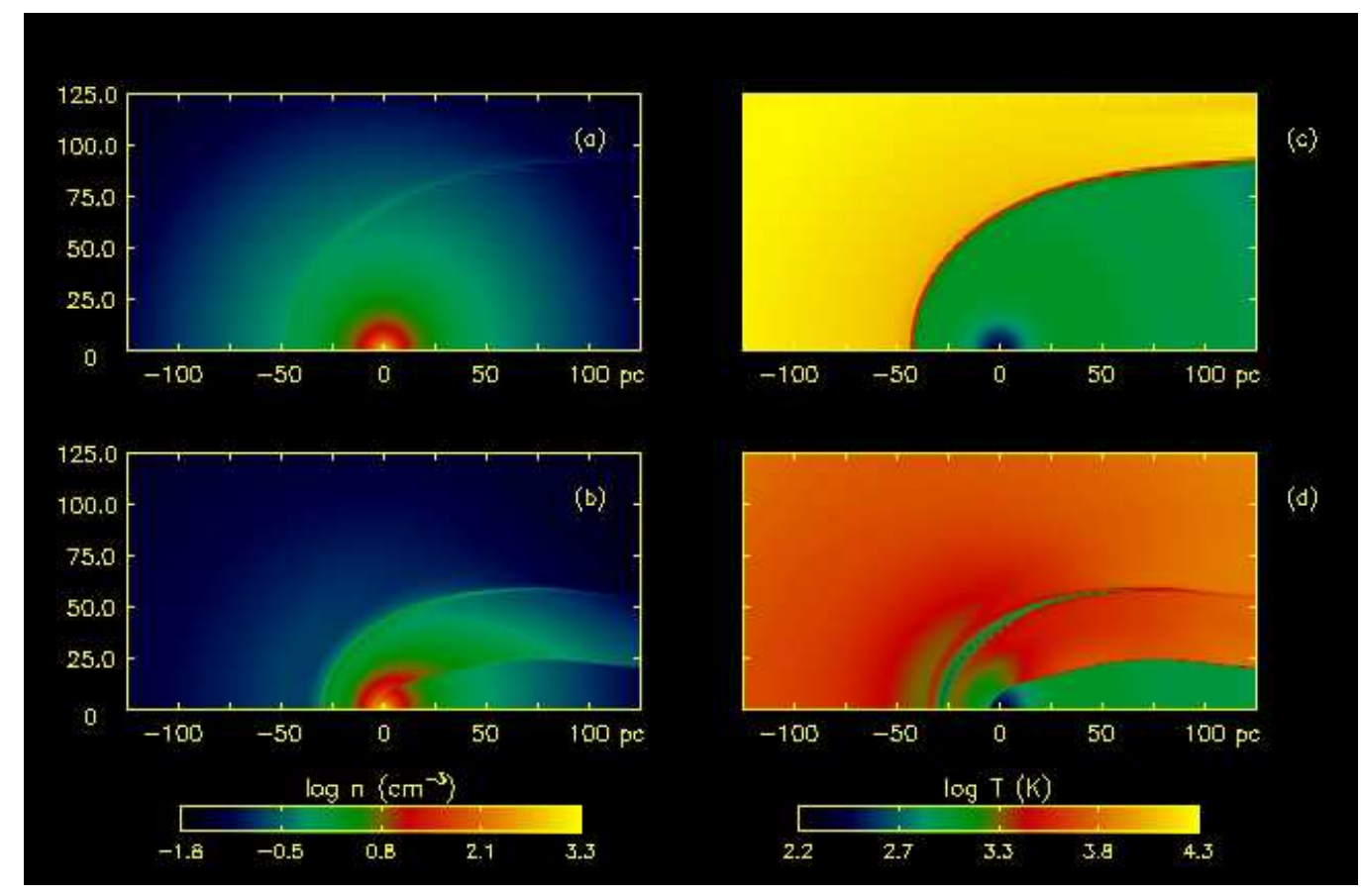

FIGURE 3. Panels (a) and (b) are density images of the 073 halo at $800 \mathrm{kyr}$ and $5.0 \mathrm{Myr}$, respectively. Panels (c) and (d) are temperatures at $800 \mathrm{kyr}$ and $5.0 \mathrm{Myr}$, respectively. 
matter was included by computing the potential necessary to cancel pressure forces everywhere on the grid (setting the halo in hydrostatic equilibrium) and holding this potential fixed throughout the simulation. The self gravity of the gas was computed every hydrodynamical time step with a two dimensional conjugate gradient Poisson solver. Cooling by electron collisional excitation and ionization, recombination, bremsstrahlung, and inverse Compton scattering of the cosmic microwave background (CMB), assuming a redshift $z=20$, was present in all the models. $\mathrm{H}_{2}$ cooling was also included using the cooling curves of Galli \& Palla [4]. For simplicity, we assume ionization and $\mathrm{H}_{2}$ fractions of $1.0 \times 10^{-4}$ and 2 $\times 10^{-6}$, respectively, appropriate for the IGM at $\mathrm{z} \sim 20$.

In Fig 2 we show profiles of ionized fraction, density, temperature, pressure, $\mathrm{H}_{2}$ fraction, and velocity along the $\mathrm{z}$-axis at three stages of photoevaporation of the 073 halo, with $\mathrm{n}_{c}=1596 \mathrm{~cm}^{-3}$ and the star $500 \mathrm{pc}$ from the center of the halo. At $200 \mathrm{kyr}$ the ionization front is R-type (becoming D-type $50 \mathrm{pc}$ from the center of the cloud). Lyman-Werner photons passing through the front partially dissociate the outer halo but the core itself remains deeply shielded and molecular hydrogen levels rise rapidly there. Panel (a) of Fig 4 shows that the core blocks LW photons in a $\sim 10$ pc band centered on the $\mathrm{z}$-axis. Central $\mathrm{H}_{2}$ fractions rise from $2 \times 10^{-6}$ to $1 \times$ $10^{-4}$ before the front becomes D-type due to catalysis by free electrons, leveling off thereafter for the lifetime of the star. The partially ionized warm gas in the outer layers of the front also catalyzes $\mathrm{H}_{2}$ production, visible as the bright yellow parabolic arc in all three panels of Fig 4. $\mathrm{H}_{2}$ fractions in the R-type front climb to $2 \times 10^{-4}$ and remain steady after the front transforms to D-type.

Density and temperature images of the D-type front at $800 \mathrm{kyr}$ and relic $\mathrm{H}$ II region at $5 \mathrm{Myr}$ are shown in Fig 3. The front has a cometary appearance because it preferentially advances in the more tenuous outer radii of the halo. Comparison of panels (a) and (b) reveals that the shadow is axially compressed by the ionized gas surrounding it but the cylindrical shock never reaches the axis in this run. Perturbations are visible in the front above the axis at $800 \mathrm{kyr}$. These fluctuations may be an early stage of instability that arises in D-type fronts when UV photons are oblique to the shock [19]. The perturbations are longer further from the central axis where radiation is incident to the front at smaller angles. LW photons preferentially stream through the underdensities, dissociating $\mathrm{H}_{2}$ beyond in bands visible between $\mathrm{z}=50 \mathrm{pc}$ and $\mathrm{z}=100 \mathrm{pc}$ at 2.29 Myr in panel (b) of Fig 4 These features are transient, vanishing by the time the star dies. The shock decelerates from $10 \mathrm{~km} / \mathrm{sec}$ at $50 \mathrm{pc}$ to 7.5 $\mathrm{km} / \mathrm{sec} 25 \mathrm{pc}$ from the core at $2.5 \mathrm{Myr}$.

When recombination commences at $2.5 \mathrm{Myr}$ molecular hydrogen forms most rapidly in the shock but then radiates outward down the density gradient in the ionized

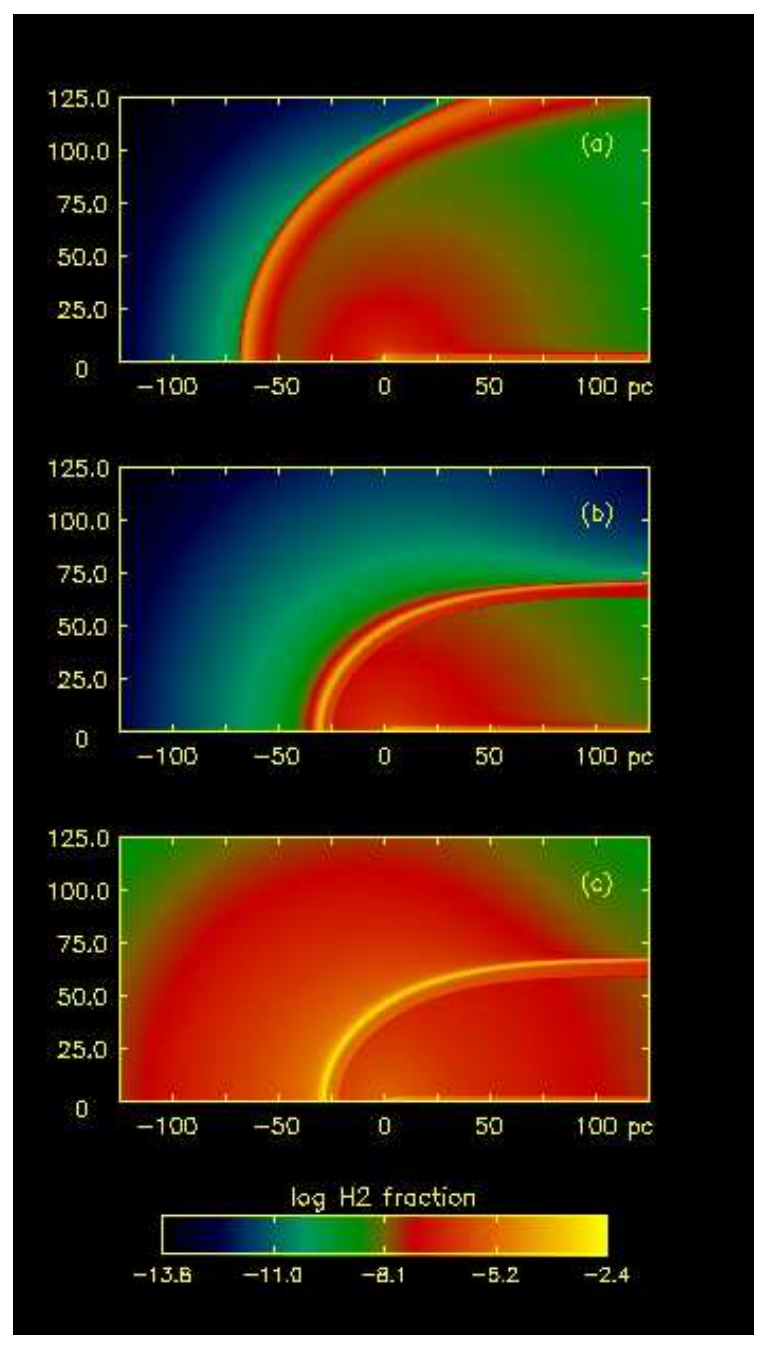

FIGURE 4. 073_500pc model: $\mathrm{H}_{2}$ fractions. Panels (a), (b) and (c) are at $210 \mathrm{kyr}, 2.29 \mathrm{Myr}$, and 2.59 Myr, respectively.

regions of the halo, as shown at 2.59 Myr in Fig $4 \mathrm{H}_{2}$ fractions rise more gradually in the core over the following 2.4 Myr, by approximately 50\%. The 5.0 Myr curves in Fig 2 show that the center of the cloud is impervious to recombination flows: the incoming shock (eventually approaching to within a few pc), the rarefaction wave, ionized backflows at larger radii, and the compression of the shadow toward the axis. One interesting feature of the relic H II region is the appearance of Rayleigh-Taylor instabilities at the interface of the warm ionized gas and shock remnant in panels (b) and (d) in Fig 3. The fingers of partially ionized (and rapidly cooling) gas lengthen along the arc away from the axis, but do not affect the dynamics of the core.

Cooling continues in the center of the halo throughout the simulation, with core temperatures falling from 200 $\mathrm{K}$ to $100 \mathrm{~K}$ and densities rising from $1596 \mathrm{~cm}^{-3}$ to 
$3000 \mathrm{~cm}^{-3}$ over 5 Myr. The shock remnant eventually ripples through the center but with only minor density fluctuations. We find that the core continues to contract while the front photoionizes the halo, reaching final densities similar to those when no star is present. Star formation proceeds unimpeded in this halo.

\section{RESULTS AND CONCLUSIONS}

Final outcomes for the other 15 models fell into four categories:

- Relatively dense halo cores are shielded from ionizing and LW UV. $\mathrm{H}_{2}$ in these cores is protected from Lyman-Werner flux even without the $\mathrm{H}_{2}$ formed in the front. Collapse of the core into a star proceeds without delay.

- In the other extreme, diffuse halos are easily destroyed by the radiation of the star wherever they reside in the cluster, either by an R-type front that ionizes the cloud on time scales much shorter than its dynamical time or by a D-type front that snowplows gas from the core at speeds greater than its escape velocity.

- Within a band of fluxes and central densities, the ionization front can jostle the core of the halo with a shock enriched with $\mathrm{H}_{2}$, compressing and speeding its collapse without destroying it. $\mathrm{H}_{2}$ is initially photodissociated in the halos but rapidly reconstitutes after the death of the star, with little delay in cooling.

- In a less forgiving sector of the flux- $\mathrm{n}_{c}$ plane the shock remnant displaces the core more violently and the implosion of the shadow deforms it more severely, but it still survives. Their effect on star formation is uncertain.

We summarize these results in Fig 5 .

\section{ACKNOWLEDGMENTS}

DW thanks Tom Abel, Kyungjin Ahn, Greg Bryan and Alex Heger for helpful discussions concerning these simulations. This work was carried out under the auspices of the National Nuclear Security Administration of the U.S. Department of Energy at Los Alamos National Laboratory under Contract No. DE-AC52-06NA25396. The simulations were performed at SDSC and NCSA under NRAC allocation MCA98N020 and at Los Alamos National Laboratory.

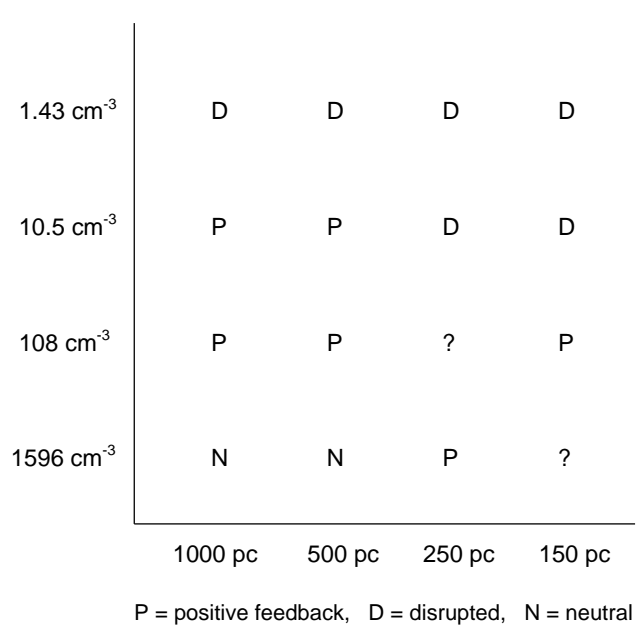

FIGURE 5. Radiative feedback on star formation in the halos.

\section{REFERENCES}

1. Abel, T., Wise, J. H., \& Bryan, G. L. 2007, ApJL, 659, L87

2. Ahn, K., \& Shapiro, P. R. 2007, MNRAS, 375, 881 ApJ, 639,621

3. Anninos, P., Zhang, Y., Abel, T., \& Norman, M. L. 1997, New Astronomy, 2, 209

4. Galli, D., \& Palla, F. 1998, A\&A, 335, 403

5. Johnson, J. L., Greif, T. H., \& Bromm, V. 2006, astro-ph/0612254

6. Machacek, M. E., Bryan, G. L., \& Abel, T. 2001, ApJ, 548, 509

7. Machacek, M. E., Bryan, G. L., \& Abel, T. 2003, MNRAS, 338, 273

8. Oh, S. P., \& Haiman, Z. 2003, MNRAS, 346, 456

9. O’Shea, B. W., Bryan, G., Bordner, J., Norman, M. L., Abel, T., Harkness, R., \& Kritsuk, A.

10. O'Shea, B. W., Abel, T., Whalen, D., \& Norman, M. L. 2005, ApJL, 628, L5

11. O'Shea, B. W., \& Norman, M. L. 2007, ApJ, 654, 66

12. Ricotti, M., Gnedin, N. Y., \& Shull, J. M. 2001, ApJ, 560, 580

13. Susa, H. 2007, ApJ, 659, 908

14. Susa, H., \& Umemura, M. 2006, ApJL, 645, L93

15. Whalen, D., \& Norman, M. L. 2006, ApJS, 162, 281

16. Whalen, D. J., \& Norman, M. L. 2007, ArXiv Astrophysics e-prints, arXiv:astro-ph/0703463

17. Whalen, D., \& Norman, M. L. 2007, ArXiv e-prints, 708, arXiv:0708.2444

18. Whalen, D., O'Shea, B. W., Smidt, J., \& Norman, M. L. 2007, ArXiv e-prints, 708, arXiv:0708.1603

19. Williams, R. J. R. 2002, MNRAS, 331, 693

20. Wise, J. H., \& Abel, T. 2007, ArXiv e-prints, 707, arXiv:0707.2059

21. Yoshida, N., Oh, S. P., Kitayama, T., \& Hernquist, L. 2006, astro-ph/0610819 